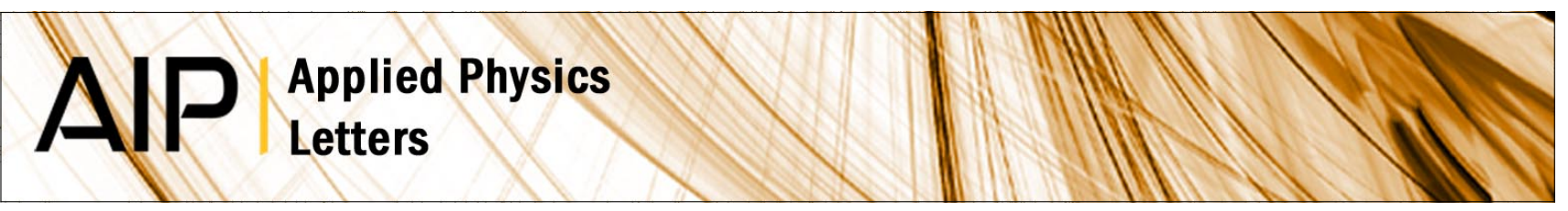

\title{
Rewritable phase-change optical recording in Ge2Sb2Te5 films induced by picosecond laser pulses
}

\author{
J. Siegel, A. Schropp, J. Solis, C. N. Afonso, and M. Wuttig
}

Citation: Appl. Phys. Lett. 84, 2250 (2004); doi: 10.1063/1.1689756

View online: http://dx.doi.org/10.1063/1.1689756

View Table of Contents: http://apl.aip.org/resource/1/APPLAB/v84/i13

Published by the American Institute of Physics.

\section{Related Articles}

Surface plasmon resonance-enabled antibacterial digital versatile discs Appl. Phys. Lett. 100, 063702 (2012)

Photoinduced write-once read-many-times memory device based on DNA biopolymer nanocomposite Appl. Phys. Lett. 99, 253301 (2011)

Photoinduced write-once read-many-times memory device based on DNA biopolymer nanocomposite APL: Org. Electron. Photonics 4, 277 (2011)

Metal-glass nanocomposite for optical storage of information Appl. Phys. Lett. 99, 201904 (2011)

Crystallization mechanisms and recording characteristics of Si/CuSi bilayer for write-once blu-ray disc Appl. Phys. Lett. 99, 121908 (2011)

\section{Additional information on Appl. Phys. Lett.}

Journal Homepage: http://apl.aip.org/

Journal Information: http://apl.aip.org/about/about_the_journal

Top downloads: http://apl.aip.org/features/most_downloaded

Information for Authors: http://apl.aip.org/authors

\section{ADVERTISEMENT}

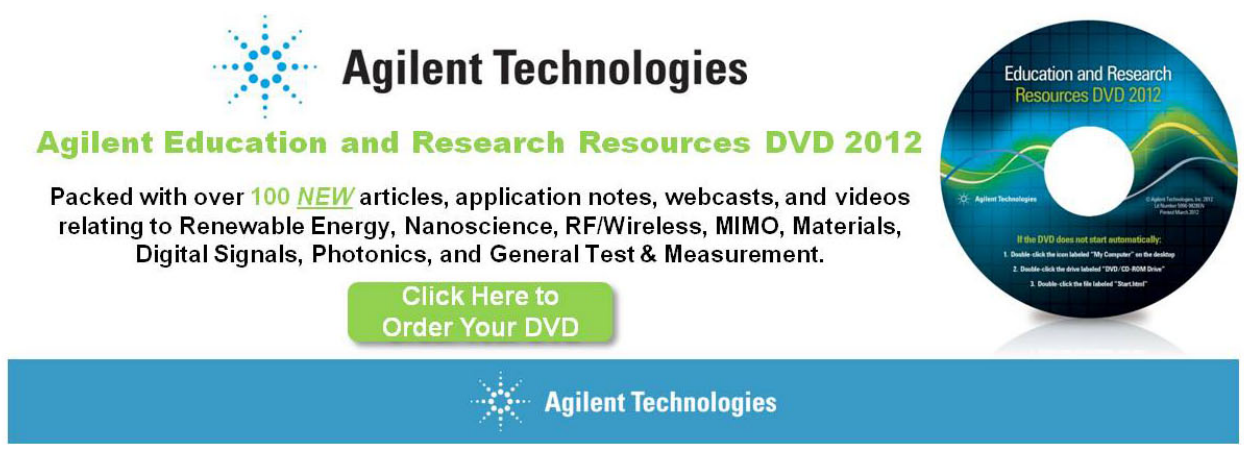




\title{
Rewritable phase-change optical recording in $\mathrm{Ge}_{2} \mathrm{Sb}_{2} \mathrm{Te}_{5}$ films induced by picosecond laser pulses
}

\author{
J. Siegel, ${ }^{\text {a) }}$ A. Schropp, ${ }^{\text {b) }}$ J. Solis, and C. N. Afonso \\ Instituto de Optica, CSIC, Calle Serrano 121, 28006 Madrid, Spain \\ M. Wuttig \\ I. Physikalisches Institut A, RWTH Aachen, 52056 Aachen, Germany
}

(Received 11 November 2003; accepted 27 January 2004)

\begin{abstract}
The phase transformation dynamics induced in $\mathrm{Ge}_{2} \mathrm{Sb}_{2} \mathrm{Te}_{5}$ films by picosecond laser pulses were studied using real-time reflectivity measurements with subnanosecond resolution. Evidence was found that the thermal diffusivity of the substrate plays a crucial role in determining the ability of the films to crystallize and amorphize. A film/substrate configuration with optimized heat flow conditions for ultrafast phase cycling with picosecond laser pulses was designed and produced. In this system, we achieved reversible phase transformations with large optical contrast ( $>20 \%)$ using single laser pulses with a duration of $30 \mathrm{ps}$ within well-defined fluence windows. The amorphization (writing) process is completed within less than $1 \mathrm{~ns}$, whereas crystallization (erasing) needs approximately $13 \mathrm{~ns}$ to be completed. (C) 2004 American Institute of Physics.
\end{abstract}

[DOI: $10.1063 / 1.1689756$ ]

Phase change optical recording is an attractive and mature technology due to the compact storage format, noncontact recording process, and long-term stability. These promising features have triggered intensive research ${ }^{1-6}$ to further improve the performance of optical discs based on GeSbTe and AgInSbTe compound films. ${ }^{7-9} \mathrm{Ge}_{2} \mathrm{Sb}_{2} \mathrm{Te}_{5}$ is referred to as a fast phase change material since both amorphization and crystallization can be triggered with laser pulses as short as $10 \mathrm{ns.}^{2,3}$ However, only a limited number of works have studied the possibility of cycling with ultrashort pulses, ${ }^{5,6,10-12}$ in spite of their potential to increase data transfer rates beyond current limits. This might be related in part to the fact that the induced phase transformations may last longer than the pulse duration. Surprisingly, this fact is mostly neglected in the relevant literature and-apart from a few works that measure the actual transformation times (Refs. 1, 4, 11-13) -most works only determine the minimum pulse duration required to induce a given phase transformation. ${ }^{2,3}$ Transformation times usually reported range between tens and hundreds of nanoseconds and may impose the limiting factor on data transfer rates in applications where rapid overwriting of existing data is required.

The only work we are aware of reporting subnanosecond transformation times is a study on $\mathrm{Ge}_{.07} \mathrm{Sb}_{.93}$ films on glass substrates exposed to single picosecond (ps) laser pulses. ${ }^{12}$ In that work, reversible phase cycling with transformation times of 400 ps for both amorphization and crystallization has been obtained. Such ultrashort transformation times were possible through a reduction of the film thickness, which effectively suppressed the recalescence effects caused by the release of latent heat during solidification. In the case of the GeSbTe system used in current compact discs, only amorphization has been achieved upon irradiation with ultrashort

\footnotetext{
${ }^{\text {a) Electronic mail: j.siegel@io.cfmac.csic.es }}$

${ }^{b)}$ Permanent affiliation: I. Physikalisches Institut A, RWTH Aachen, 52056 Aachen, Germany.
}

laser pulses. ${ }^{6}$ The goal of this letter is to achieve phase reversibility in $\mathrm{Ge}_{2} \mathrm{Sb}_{2} \mathrm{Te}_{5}$ upon ps laser pulse irradiation and thus increase the data transfer rates of both processes, data storage and data overwriting. In the present work we have followed an approach different from that used earlier ${ }^{12}$ for $\mathrm{Ge}_{.07} \mathrm{Sb}_{.93}$ to address the latent heat issue. Instead of reducing the film thickness-and thus sacrificing a potential high optical contrast-we have modified in a controlled way the heat extraction toward the substrate.

Thin $\mathrm{Ge}_{2} \mathrm{Sb}_{2} \mathrm{Te}_{5}$ films were deposited by dc-magnetron sputtering in a vacuum chamber held in an $\mathrm{Ar}$ atmosphere of $10^{-1} \mathrm{~Pa}$, using a stoichiometric target. Films were deposited on a range of different substrates, including glass slides and multilayer substrates consisting of a glass slide onto which a 100-nm-thick aluminum layer was deposited, followed by a dielectric layer of $\mathrm{ZnS}-\mathrm{SiO}_{2}$ of varying thickness $d$, with $d=15,25$ and $50 \mathrm{~nm}$. On top of these substrates, the optical recording layer $\left(\mathrm{Ge}_{2} \mathrm{Sb}_{2} \mathrm{Te}_{5}\right)$ with a constant thickness of 50 $\mathrm{nm}$ was deposited. Details of the deposition conditions and composition analysis can be found in Ref. 2. In order to initialize the as-deposited amorphous samples, a row of 0.5mm-diam spots were precrystallized by exposure to $\approx 30$ consecutive ps laser pulses generated by an amplified dye $\operatorname{laser}^{14}$ (30 ps pulse duration, $583 \mathrm{~nm}$ wavelength) at energies well below the melt threshold, thus inducing solid-state crystallization. The initialized spots were then irradiated with single laser pulses to induce amorphization. Once an irradiated spot was successfully amorphized, the same spot was irradiated with another single laser pulse to induce crystallization. In each case, the dynamics of the phase transformations were followed in real time by measuring the evolution of the reflectivity. A detailed description of the setup for transient reflectivity measurements with subnanosecond resolution can be found elsewhere. ${ }^{14}$ Essentially, it consisted of a continuous wave probe beam [a HeNe laser at $632.8 \mathrm{~nm}$ for the results in Figs. 1(a) and 1(b) and a single-mode 


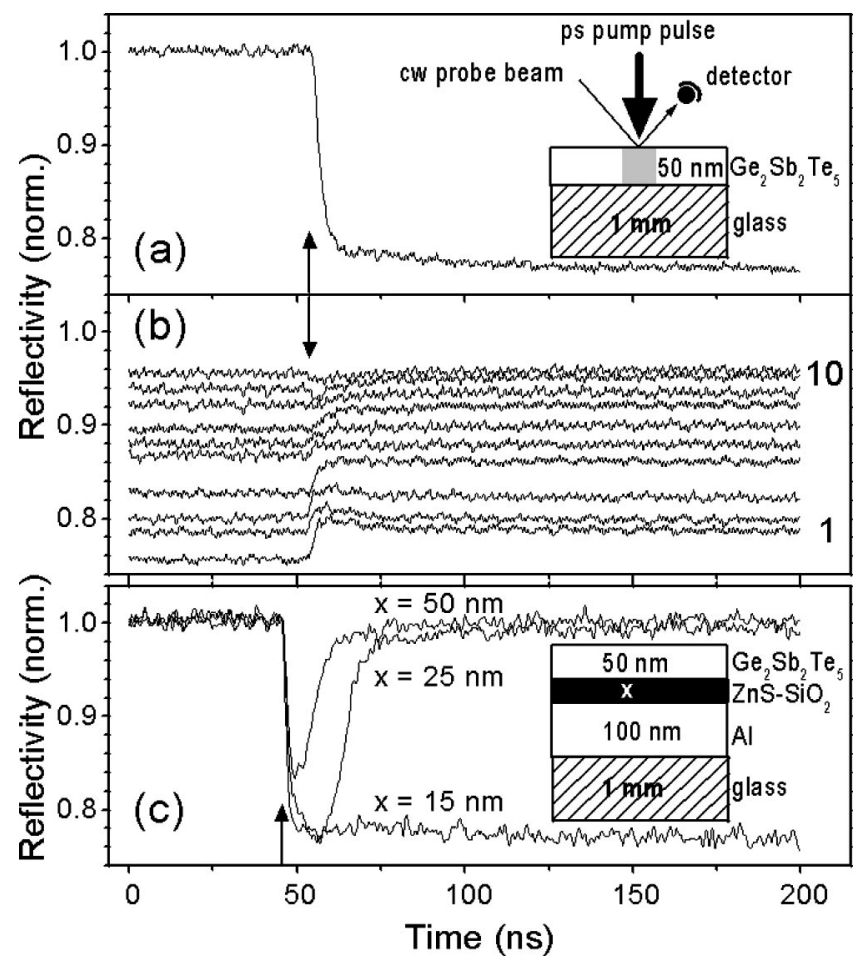

FIG. 1. The inset in (a) shows the principle of real-time reflectivity measurements in $50 \mathrm{~nm} \mathrm{Ge} \mathrm{Sb}_{2} \mathrm{Te}_{5}$ films on glass substrate. (a) Reflectivity evolution during single ps laser pulse induced amorphization. (b) Reflectivity evolution during gradual crystallization by repetitive ps pulse irradiation of the same spot amorphized in (a) (pulses 1-10 are shown). (c) Reflectivity evolution during single ps laser pulse induced amorphization attempts in 50 $\mathrm{nm} \mathrm{Ge}_{2} \mathrm{Sb}_{2} \mathrm{Te}_{5}$ films on $\mathrm{ZnS}-\mathrm{SiO}_{2} / \mathrm{Al} /$ glass [see the inset in (c)]. Each transient corresponds to a different thickness of the heat buffer layer $\mathrm{ZnS}-\mathrm{SiO}_{2}$. The fluences used to obtain the transients are: (a) $90 \mathrm{~mJ} / \mathrm{cm}^{2}$, (b) $23 \mathrm{~mJ} / \mathrm{cm}^{2}$, (c) $31 \mathrm{~mJ} / \mathrm{cm}^{2}$ for $x=50 \mathrm{~nm}, 47 \mathrm{~mJ} / \mathrm{cm}^{2}$ for $x=25 \mathrm{~nm}$, and $52 \mathrm{~mJ} / \mathrm{cm}^{2}$ for $x=15 \mathrm{~nm}$. All transients were acquired with a fast photodiode and were normalized to the reflectivity of the crystalline phase. The arrows indicate the temporal position of the laser pulse.

$\mathrm{Ar}^{+}$-ion laser at $514.5 \mathrm{~nm}$ for the results in Fig. 1(c) and Figs. 2(a) $-2(d)]$ incident at the sample surface with $s$ polarization at an angle of $\sim 18^{\circ}$ and focused down to a spot size of $30 \mu \mathrm{m}$ at the center of the area irradiated with the ps pump beam ( $240 \mu \mathrm{m}$ diameter). Two detection systems were used in parallel to ensure both a large time window and high temporal resolution. We used a fast photodiode and a fast oscilloscope providing a variable time window with a temporal resolution of a few nanoseconds (ns). A streak camera in single-sweep mode was used providing a time window of about $50 \mathrm{~ns}$ and a temporal resolution of $350 \mathrm{ps}$.

Figure 1(a) shows the dynamics of the amorphization process of a precrystallized area of a film on glass, induced by a single ps pulse. Within the time resolution used, the reflectivity decreases from its high initial level (the reflectivity values are normalized to the reflectivity of the precrystallized spot) down to a value below 0.8 , yielding a reflectivity contrast $>20 \%$. This process is generally understood to occur through complete melting and rapid solidification of the film, at quenching rates that are sufficiently fast to prevent the formation of the crystalline phase. ${ }^{3}$ However, the inverse transformation, i.e., recrystallization of the amorphized spot, cannot be triggered by a single laser pulse in this sample. Figure 1(b) shows a sequence of transients obtained by repetitive irradiation of the previously amorphized region at

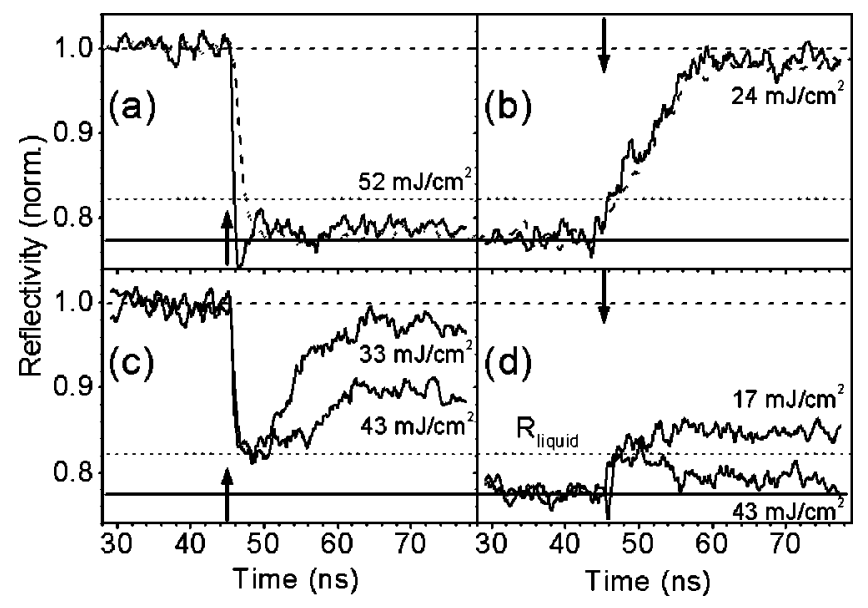

FIG. 2. (a) Reflectivity evolution during single ps laser pulse induced amorphization and (b) crystallization in $50 \mathrm{~nm} \mathrm{Ge}_{2} \mathrm{Sb}_{2} \mathrm{Te}_{5}$ on $15 \mathrm{~nm}$ $\mathrm{ZnS}-\mathrm{SiO}_{2} / 100 \mathrm{~nm} \mathrm{Al} /$ glass, as measured with a fast photodiode (dotted curves) and a Streak Camera (solid curves). (c) Amorphization attempts and (d) crystallization attempts with nonoptimized fluence values in the same sample as measured with the Streak Camera. The dashed line corresponds to the reflectivity level of the crystalline phase, the solid line to the level of the amorphous phase, and the dotted line to the level of liquid phase $R_{\text {liquid }}$. The arrows indicate the temporal position of the laser pulse.

constant fluence, leading to a gradual increase (partial crystallization) of the reflectivity level.

The inability of this sample to crystallize upon single pulse irradiation is related to the short laser pulse duration and the heat flow conditions. The use of ps laser pulses leads to a situation in which the pulse energy is deposited within a very short time in a thin surface layer, leading to heating or melting. The so-generated steep temperature gradient is subsequently smoothed by heat diffusion towards the substrate. The characteristic diffusion length $L_{\text {th }}$ during the pulse duration $\tau$ depends on the thermal diffusivities $D$ of both the film and the underlying substrate according to $L_{\mathrm{th}} \approx(D \tau)^{1 / 2}$. Essentially, the diffusion length during the ps pulse is negligible $\left(L_{\text {th }} \approx 4 \mathrm{~nm}\right.$ for $\tau=30 \mathrm{ps}$ and $D\left(\mathrm{Ge}_{2} \mathrm{Sb}_{2} \mathrm{Te}_{5}\right)=0.0045$ $\mathrm{cm}^{2} / \mathrm{s}$-Refs. 6 and 15), whereas it would exceed the film thickness when using ns laser pulses as commonly done in phase change optical recording.

In order to achieve single pulse crystallization by ps laser pulses we have selected the thermal diffusivity of the substrate to be lower than that of glass ( $D=0.008 \mathrm{~cm}^{2} / \mathrm{s}$-Ref. 6). This leads to an increase of the film temperature and a reduction of the temperature gradient. We have used a layered substrate composed of a layer of high thermal diffusivity (Al, $D=0.88 \mathrm{~cm}^{2} / \mathrm{s}$-Ref. 6) used as a heat sink and an intermediate layer of very low thermal conductivity acting as a heat buffer $\left(\mathrm{ZnS}-\mathrm{SiO}_{2}, \quad D\right.$ $=0.0032 \mathrm{~cm}^{2} / \mathrm{s}$-Refs. 6 and 15). By varying the thickness of the $\mathrm{ZnS}-\mathrm{SiO}_{2}$ layer, the effective thermal diffusivity of the layered substrate can be adjusted between the values of the heat sink and the heat buffer.

Figure 1(c) shows reflectivity transients corresponding to amorphization attempts in films with $\mathrm{ZnS}-\mathrm{SiO}_{2}$ buffer layers of different thicknesses. We have selected for each thickness $x$ the transient that has been obtained at a fluence that came closest to amorphization. For $x=50 \mathrm{~nm}$, one can appreciate a rapid reflectivity decrease related to melting that is immediately followed by a rapid complete recovery due to recrystallization. We attribute the inability of these films to amor- 
phize to a too low effective thermal diffusivity of the multilayer substrate system, which prevents the molten film from achieving an undercooling sufficiently large for amorphization. For $x=25-\mathrm{nm}$, recrystallization occurred somewhat later but again it was not possible to amorphize the films. However, for $x=15-\mathrm{nm}$, we have been able to induce amorphization of a precrystallized spot with a single ps laser pulse, confirming that the reduction of the buffer layer thickness increases the heat flow to the substrate.

Figure 2(a) shows again, on a shorter time scale, the successful amorphization in the sample with a buffer layer thickness of $15 \mathrm{~nm}$. By exposing the so-amorphized spot to a single ps laser pulse of lower energy, we have also been able to induce complete recrystallization [Fig. 2(b)]. Both transformations have been shown to be stable, reversible and repeatable, exhibiting an optical contrast $>20 \%$. The amorphization and crystallization process is completed within less than 1 and $13 \mathrm{~ns}$, respectively [Figs. 2(a) and 2(b)]. It is worth pointing out that it is indeed necessary to make use of a Streak Camera for determining the transformation times, since the amorphization time would appear longer when measured with a detection system with lower resolution [as shown by the dashed curve in Fig. 2(a)].

The results shown in Figs. 2(a) and 2(b) clearly demonstrate that the 15-nm-thick buffer layer in combination with a thick Al layer provides a good compromise in terms of heat flow for ps laser induced phase cycling in $\mathrm{Ge}_{2} \mathrm{Sb}_{2} \mathrm{Te}_{5}$ films. The heat extraction upon complete melting is sufficiently fast to achieve the undercooling necessary for amorphization to take place, but it is also sufficiently slow at low fluences to promote complete crystallization. The fluence windows to obtain phase cycling with short transformation times are narrow as demonstrated in Figs. 2(c) and 2(d), showing, respectively, unsuccessful amorphization and crystallization attempts at different fluences. For attempted amorphization of the crystalline phase at low fluences [Fig. 2(c)] the undercooling obtained is too low and partial or complete recrystallization is induced. For attempted crystallization of the amorphous phase at too low fluences $\left[17 \mathrm{~mJ} / \mathrm{cm}^{2}\right.$, Fig. 2(d)] only partial crystallization is obtained. Interestingly, for attempted crystallization at too high fluences $\left[43 \mathrm{~mJ} / \mathrm{cm}^{2}\right.$, Fig. 2(d)] - which is considerably above the fluence for singlepulse crystallization [Fig. 2(b)] - the spot melts initially (reflectivity increase) but reamorphizes completely afterwards (reflectivity decrease to the initial level). We attribute this behavior at high fluences to an increased melt depth and therefore to a more efficient heat extraction toward the substrate, favoring re-amorphization. Essentially, it is the same process of solidification as observed in Fig. 2(a), where amorphization is observed at high fluences, but starting from the crystalline phase. An important conclusion is, therefore, that the amorphization process induced by a single ps laser pulse in this sample is independent of the initial phase.

There are strong indications that the crystallization process also involves surface melting. The transients shown in Fig. 2(c) have a common reflectivity minimum of approximately 0.82 that we interpret as the reflectivity of the liquid phase $R_{\text {liquid }}$. Approximately the same reflectivity value is obtained in the transients in Fig. 2(d) at the initial reflectivity rise after the laser pulse. Since the transients in Fig. 2(d) correspond to fluences below and above the optimum crystallization fluence [Fig. 2(b)], we conclude that surface melting should also occur in the latter case. Unfortunately, the occurrence of melting cannot be resolved in Fig. 2(b) due to the fluctuations of the probe laser beam (noise).

In conclusion, we have been able to achieve reversible and stable phase transformations with an optical contrast $>20 \%$ in $\mathrm{Ge}_{2} \mathrm{Sb}_{2} \mathrm{Te}_{5}$ films upon irradiation with single laser pulses of $30 \mathrm{ps}$. The use of laser pulses of $30 \mathrm{ps}$ combined with the achievement of fast transformation times $(<1 \mathrm{~ns}$ for amorphization and $13 \mathrm{~ns}$ for crystallization) clearly demonstrates the potential increase in the data transfer rates for both processes, data storage $(>5 \mathrm{Gbit} / \mathrm{s})$ and data overwriting ( $>11 \mathrm{Mbit} / \mathrm{s}$ ). Phase cycling and high transformation speeds have been achieved through careful adjustment of the heat extraction toward the substrate. The best performance was obtained for the $50 \mathrm{~nm} \mathrm{Ge}{ }_{2} \mathrm{Sb}_{2} \mathrm{Te}_{5} / 15 \mathrm{~nm} \mathrm{ZnS}-\mathrm{SiO}_{2} / 100 \mathrm{~nm}$ $\mathrm{Al} /$ glass system. Recently developed compact diode lasers emitting pulses of $60 \mathrm{ps}$ at high repetition rates (up to $40 \mathrm{MHz}$ ) and short wavelengths (down to $375 \mathrm{~nm}$ ) have already found widespread applications in fluorescence spectroscopy ${ }^{16}$ and could provide an ideal laser source for high data transfer optical recording.

H. Dieker and S. Ziegler (RWTH Aachen) are thanked for producing the samples. This work has been partially supported by the EU in the frame of the TMR Project XPOSE (Grant No. HPRN-CT-2000-00160).

${ }^{1}$ J. Carriere, R. Narayan, W.-H. Yeh, C. Peng, P. Khulbe, L. Li, R. Anderson, J. Choi, and M. Mansuripur, Principles of Optical Disk Data Storage, in Progress in Optics Vol. 41, edited by E. Wolf (Elsevier Science, New York, 2000), p. 2, and references therein.

${ }^{2}$ V. Weidenhof, I. Friedrich, S. Ziegler, and M. Wuttig, J. Appl. Phys. 89, 3168 (2001).

${ }^{3}$ V. Weidenhof, N. Pirch, I. Friedrich, S. Ziegler, and M. Wuttig, J. Appl. Phys. 88, 657 (2000).

${ }^{4}$ P. K. Khulbe, E. M. Wright, and M. Mansuripur, J. Appl. Phys. 88, 3926 (2000)

5 J. Solis and C. N. Afonso, Appl. Phys. A: Mater. Sci. Process. 76, 331 (2003)

${ }^{6}$ T. Ohta, N. Yamada, H. Yamamoto, T. Mitsuyu, T. Kozaki, J. Qiu, and K. Hirao, Mater. Res. Soc. Symp. Proc. 674, V1.1.1 (2001).

${ }^{7}$ H. Iwasaki, Y. Ide, M. Harigaya, Y. Kageyama, and I. Fujimura, Jpn. J. Appl. Phys., Part 1 31, 461 (1992).

${ }^{8}$ G. F. Zhou and B. A. J. Jacobs, Jpn. J. Appl. Phys., Part 1 38, 1625 (1999).

${ }^{9}$ H. J. Borg, P. M. M. Blom, B. A. J. Jacobs, B. Tieke, A. E. Wilson, I. P. D. Ubbens, and G. F. Zhou, Proc. SPIE 3864, 191 (1999).

${ }^{10}$ C. N. Afonso, J. Solis, F. Catalina, and C. Kalpouzos, Appl. Phys. Lett. 60, 3123 (1992)

${ }^{11}$ M. C. Morilla, J. Solis, and C. N. Afonso, Jpn. J. Appl. Phys., Part 1 36, 1015 (1997)

${ }^{12}$ J. Siegel, C. N. Afonso, and J. Solis, Appl. Phys. Lett. 75, 3102 (1999).

${ }^{13}$ J. Wei and F. Gan, Thin Solid Films 441, 292 (2003).

${ }^{14}$ J. Solis, J. Siegel, and C. N. Afonso, Rev. Sci. Instrum. 71, 1595 (2000).

${ }^{15}$ R. Zhao, K. Lim, J. Liu, J. Ho, T. Chong, Z. Liu, B. Xu, and L. Shi, Jpn. J. Appl. Phys., Part 1 39, 3458 (2000).

${ }^{16}$ D. S. Elson, J. Siegel, S. E. D. Webb, S. Lévêque-Fort, M. J. Lever, P. M. W. French, K. Lauritsen, M. Wahl, and R. Erdmann, Opt. Lett. 27, 1409 (2002). See also PicoQuant-website: http://www.picoquant.de/ 